\title{
The Research to Action Project: Applied Workplace Solutions for Nurses
}

Linda Silas RN, BScN

President, Canadian Federation of Nurses Unions

\section{Abstract}

The number of new nurses entering the profession has increased, but the need to retain nurses in the profession continues to be a critical priority. The consequences of the nursing shortage are reflected in continued high levels of overtime, absenteeism and turnover.

The Canadian Federation of Nurses Unions (CFNU), in partnership with the Canadian Nurses Association, the Canadian Healthcare Association and the Dietitians of Canada, initiated the project Research to Action: Applied Workplace Solutions for Nurses (RTA). The RTA initiative comprised research-based pilot projects, implemented in 10 jurisdictions across the country, that aimed to improve workplaces and increase the retention and recruitment of nurses. Unions, employers, governments, universities and professional associations came together in an unprecedented show of collaboration. Lessons and knowledge were shared among the projects, which were evaluated for their viability in other jurisdictions and professions. The pilots led to increased leadership, engagement and professional development, and decreased overtime, absenteeism and turnover.

\section{Background}

Although nurses constitute one-third of the Canadian healthcare workforce, there continues to be a pervasive shortage of nursing human resources. The Canadian Nurses Association predicts a shortfall of 60,000 full-time equivalents (FTEs) by 2022 (Tomblin Murphy et al. 2009). Governments and health organizations have implemented a number of strategies to increase the supply of nurses, with varying degrees of success. Nursing research shows that working conditions and the workplace environment are significant factors in the struggle to retain nurses. Registered nurses and nurse supervisors who were publicly employed in 
the Health Care and Social Assistance sector worked a total of 20,627,800 hours of overtime in 2010 - the equivalent of 11,400 full-time jobs, at a cost of $\$ 891$ million annually. That same year, an average of 19,200 publicly employed nurses were absent from work each week because of illness or disability (CFNU 2011). Research shows that nurses have one of the highest rates of absence due to illness or disability compared with other occupations (Lasota 2009).

Nurse burnout and job dissatisfaction, precursors of voluntary turnover, also increase significantly as workload increases (Aiken et al. 2002). The turnover of nurses is a major problem in Canadian hospitals. A 2008 study on the costs and outcomes of nurse turnover in Canadian hospitals found that the average nursing turnover rate is close to $20 \%$ per year, at an average cost of $\$ 25,000$ per nurse (O’Brien-Pallas et al. 2008).

The Canadian nursing shortage, its underlying causes and its potential to become dramatically worse have been documented by a wealth of studies over the past number of years. Several innovative suggestions to address these issues have surfaced in the academic literature, but little has been done to bridge the gap between research and practice. We continue to witness high levels of nurse overtime, sick time and a frustrated profession, all of which have a negative impact on patient care. Clearly, workplace culture needs to be reformed, and this reform must involve front-line nurses.

The CFNU wanted to begin this process of culture change by applying some of the innovative approaches advocated in research. In 2006, with funding from Human Resources and Skills Development Canada, the CFNU successfully implemented two pilot projects: Critical Care and Emergency Nursing Programs in Cape Breton, Nova Scotia and a mentorship program in the Regina Qu'Appelle Health Region in Saskatchewan. The success of these projects encouraged the CFNU to plan a more ambitious undertaking.

Early in 2008, the CFNU worked with its nine provincial nurses' unions, local employers and provincial/territorial governments to develop workplace projects that would promote positive healthcare workplaces. The proposals were premised on the conviction that workplace reform requires the collaboration of employers, governments, unions and other key stakeholders. These partners were required to provide concrete support, either financially or in kind. The objective was to bring research into action - to take research off the shelf and bring it to life in nursing workplaces across the country. 
The project proposals were completed by mid-July 2008. They identified potential partners, the type of project and methodology (e.g., mentoring program, nurse-to-patient ratios, 80/20 model for staffing), the scope, objectives and deliverables, the proposed timeframe, an evaluation plan and a preliminary budget, including the proposed contributions from each partner. All draft proposals were reviewed and approved by the identified partners in each jurisdiction.

\section{Overview}

In October 2008, Health Canada approved a proposal by the CFNU to implement pilot projects aimed at improving nurse retention and recruitment through various workplace improvement strategies. The four core objectives of the projects were to

1.implement innovative, research-based strategies (pilot projects) and evaluate their impact on the retention and recruitment of nurses;

2. engage nurses' unions, employers, governments, educators and other healthcare stakeholders in collaborative partnerships;

3. develop resources that build capacity within the workplace; and

4. share and transfer knowledge within and across jurisdictions and professions.

Collectively, the 10 pilots were known as Research to Action: Applied Workplace Solutions for Nurses (RTA). Each pilot focused on an aspect of nursing practice that had been identified as particularly relevant to the jurisdiction.

The pilots involved the implementation of innovative, research-based strategies to enhance the quality of patient care by addressing staffing issues, to offer support to new nursing graduates and newly hired nurses, and to provide opportunities for education and professional development. All projects were workplace based and developed in partnership with employers, unions, governments and other healthcare stakeholders in each jurisdiction. Each pilot was led by its own provincial/territorial steering committee that included the provincial/territorial department of health, the provincial nurses' union, the employer (usually a hospital or regional health authority) and, in some cases, representation from an academic institution, professional association or both. Each steering committee hired its own project coordinator and additional support staff as required.

A national steering committee with membership from the CFNU, its national partners (the Canadian Nurses Association, the Canadian Healthcare Association and the Dietitians of Canada) and two representatives from each pilot project helped oversee the implementation of the project. The Fédération interprofes- 
sionnelle de la santé du Québec and Health Canada participated on the national steering committee on an ex-officio basis. A national management team (NMT), guided by the president of the CFNU, provided day-to-day management of the RTA projects. The members of the NMT offered training, guidance, advice and support to the 10 project coordinators on an ongoing basis by means of monthly conference calls, the use of common project management software to help keep track of the projects (progress reports, conference planning and so on) and four in-person meetings over the course of the projects. The two leads of the NMT and the president of the CFNU made a total of 38 site visits to the pilots. This contact enabled a first-hand understanding of each project and provided the opportunity to offer guidance and contribute to knowledge transfer.

\section{The projects}

With an eye to the four objectives mentioned above, the pilots considered ways to

- infuse workplaces with improved morale;

- create sustainable, ongoing programs supported by specific tools and resources;

- help nurses develop new skills and expertise; and

- create strategies and programs that could be transferable to different jurisdictions and health professions.

The following list offers a snapshot of the 10 pilot projects, each of which is discussed in greater detail in the papers that follow:

- British Columbia: An 80/20 staffing model was implemented for nurses working on a unit of an acute care facility in a smaller interior city.

- Alberta: The project evaluated the impact of seven nurse retention and recruitment initiatives implemented in the province in recent years.

- Saskatchewan: The Synergy staffing tool (Curley 2007) was applied on an acute care unit at St. Paul's Hospital in Saskatoon. The tool is designed to facilitate nurse staffing decisions based on the needs of patients (nurse-to-patient ratios).

- Manitoba: An enhanced orientation and mentorship program for nurses new to long-term care was implemented at three facilities in Winnipeg.

- Ontario: A dashboard staffing tool was implemented on eight acute care units across Hamilton Health Sciences. The dashboard is meant to encourage nurses to become more engaged in staffing decisions based on the needs of patients and available resources.

- New Brunswick: The project included a French-language, online orientation program for newly hired nurses as well as a mentoring program linking senior nurses with new recruits. 
- Nova Scotia: The project made use of an 80/20 staffing model to provide mentorship workshops and to implement a mentorship program linking senior nurses with new hires. The project also enhanced a web-based program called HSPnet that will help new nursing graduates find jobs in the province.

- Prince Edward Island: Critical care and emergency nursing programs were introduced in the province so that nurses no longer needed to travel to Halifax to take the 13- to 15-week courses offered there.

- Newfoundland and Labrador: The project implemented an 80/20 staffing model for nurses in a small long-term care facility in a rural setting.

- Nunavut: The project provided professional development in emergency and critical care as well as mentorship opportunities to nurses working at the largest acute care facility in the territory.

\section{Evaluation}

Tomblin Murphy Consulting was contracted to conduct a national evaluation of the RTA pilot projects. The evaluation used an Outcome Mapping (OM) methodology with a mixed-methods, repeat survey (before/after) study design. In two jurisdictions, Alberta and Nunavut, project evaluations were coordinated by the RTA national management team but conducted apart from the national evaluation. The Alberta project consisted of a large-scale evaluation of retention and recruitment initiatives contained in the collective agreement; the scope and depth of that project made it expedient to conduct a separate evaluation. The Nunavut project started 11 months after the other projects, at which point the national evaluation was well underway. It was thus necessary to conduct a separate evaluation.

The projects in British Columbia, Saskatchewan and Ontario chose to conduct supplementary provincial evaluation reports to gather additional information. The national evaluation consultants worked with these jurisdictions to coordinate activities to minimize duplication.

\section{Summary of RTA Results}

The overall goal of the Research to Action initiative was to test innovative strategies that would improve retention and recruitment of nurses, promote highquality work environments, develop collaborative partnerships and facilitate knowledge transfer.

1. Innovative strategies implemented to improve the retention and recruitment of nurses

The 10 projects utilized existing nursing research that was adapted to respond to local needs and conditions. The RTA initiative provided a multitude of different 
training and professional development opportunities. For example, 42 nurses participated in the 80/20 professional development staffing model, 28 upgraded skills through courses in such areas as critical care training, 91 used and evaluated staffing tools, more than 140 benefited from a mentorship or preceptorship relationship and over 500 participated in workshops. In total, nurses were engaged on 1,737 occasions. Other health professionals also participated in and benefited from specific project activities.

Participating nurses and project partners reported that they were engaged by the projects and motivated by a shared vision for the nursing workplace. They recognized the importance of a healthy workplace for the retention and recruitment of nurses and the value of the collaborative model for achieving these ends.

\section{New collaborative partnerships}

The entire RTA initiative involved more than 60 partners at the national, provincial/territorial and local levels and demonstrated that nurses' unions, employers, governments, academics and others can successfully engage in collaborative partnerships to address specific nursing workforce issues. The structure of the projects helped partners to better understand other stakeholders. Partners came to understand that if they really wished to make changes, all stakeholders had to assume joint responsibility. In the end, project partners provided $\$ 26.74$ million in financial and in-kind support on top of the $\$ 4.7$ million in funding provided by Health Canada.

The evaluation found that project partners benefited from the collaborative relationships and indicated a desire to continue with these partnerships. The improved working relationships among different stakeholder groups form a basis of mutual trust and goodwill that will benefit future projects. Workplace culture change can be achieved only over the long term, and thus the continuation of collaborative efforts is essential.

3. Increased capacity within the workplace

Building capacity was a key component of the Research to Action initiative. For example, it

- supported the addition of valuable infrastructure, such as the patient simulators purchased for Nunavut and PEI (tools and equipment purchased for the projects will remain in the jurisdictions to support the continuation of the programs); 
- developed workshop materials in both French and English that are now being shared across jurisdictions and provinces/territories;

- provided mentorship programs in four jurisdictions and trained more than 140 mentors and protégés;

- provided workshops and other training for more than 500 nurses;

- provided opportunities for nurses to upgrade their skills and acquire new competencies (for example, 26 nurses in Nunavut received critical care training, and 16 nurses in PEI received certification after completing emergency and critical care training programs);

- increased the capacity of jurisdictions to deliver training programs on site, such as the PEI critical care and emergency nursing courses;

- increased capacity for project management and research (individual project coordinators developed new skills that have benefited their employers);

- supported the development of policy and guidance documents, as well as materials for workshops and new tools; and

- produced significant data on retention and recruitment initiatives, via the Alberta project and the overall RTA initiative.

\section{Knowledge transfer within and across jurisdictions and professions}

One of the four primary goals of the RTA initiative was to facilitate knowledge transfer (KT) within and across jurisdictions and professions. KT activities included

- 15 project coordinator and steering committee member visits to RTA project sites;

- six presentations and three poster presentations at national conferences;

- presentations at the International Council of Nurses convention in South Africa (2009) and Malta (2011);

- 14 presentations at provincial/territorial conferences;

- 103 meetings/presentations to provincial/territorial organizations, groups and media;

- 24 monthly teleconferences with project coordinators;

- the production of one national and 10 provincial/territorial videos on the RTA initiative (available online);

- a project management site that acted as a portal for information sharing; and

- a website hosted on the CFNU's Think Nursing website (www.thinknursing. $\mathrm{ca} / \mathrm{rta}$ ) that includes unique pages for each project with overviews, documents and videos. 
A Knowledge Transfer Conference held in March 2011 presented the results of the 10 initiatives to a broader audience. More than 100 participants attended, representing front-line nurses, employers, unions, academics and provincial, territorial and federal governments.

\section{Evaluation Summary}

The overall goal of the Research to Action initiative was to test innovative strategies that would improve retention and recruitment of nurses, promote highquality work environments, develop collaborative partnerships and facilitate knowledge transfer.

The results far exceeded initial expectations:

- Nurses were engaged on at least 1,737 occasions.

- Over 500 nurses participated in orientation and mentorship workshops, and more than 140 nurses were involved in mentorship relationships.

- Job satisfaction improved and nurses reported greater empowerment and occupational commitment.

- The number of nurses reporting a high level of leadership and support rose $147 \%$.

- There was a 10\% decrease in turnover, overtime and absenteeism from baseline to follow-up.

- There was a $66.7 \%$ increase in time dedicated to professional development.

- More than 60 stakeholders, including employers, governments and unions, developed new working relationships at the national, provincial and local levels.

- There were more than 140 unique events to promote knowledge transfer within and across jurisdictions and professions.

- Nursing capacity in workplaces and regions increased, with new infrastructure, resources, tools and skills.

\section{Lessons Learned}

1. Focus on the workplace

In order to improve retention and recruitment of nurses, the workplace culture has to change. Real change has to come from the bottom up. The Research to Action initiative focused on the front-line nurses who can make this happen. Involving staff nurses in research, policy and the overall project oversight has in no small way contributed to the success of the RTA initiative.

2. Ongoing commitment to professional development and skill acquisition is an essential component in improving retention and recruitment 
The RTA initiative offered nurses an opportunity to acquire new skills and competencies and to share what they learned with their colleagues. This commitment to professional development has led to improved morale and renewed professional commitment. Nurses know that resources are scarce, but it is essential that they see there is a commitment to support this high-priority area.

\section{Sharing of knowledge}

The RTA initiative demonstrated the importance of sharing knowledge and lessons from the individual projects with a broad audience. There are several examples of programs developed in one jurisdiction and then modified and adapted for use in another region. By involving members of the academic community in several projects, the RTA initiative also helped to increase communication and understanding between educators and health professionals.

\section{Partnerships are critical to success and sustainability}

The RTA initiative has demonstrated that fixing the workplace is a joint responsibility. Partners need to contribute resources to implementing solutions. The collaborative approach involving all key stakeholders can be time-consuming. However, engagement of all partners means there will be ongoing support and a willingness to find solutions when challenges arise.

\section{Planning and transparency are important}

If real change is to occur, there has to be time to plan realistically and to include all the relevant individuals, including front-line staff. Project costs must be realistically estimated to avoid surprises.

\section{Provide necessary support and resources}

The RTA initiative provided a local project coordinator for each of the 10 projects. These individuals were hired by the local steering committees and were based in the project workplace. They provided support to participants, were able to troubleshoot when difficulties arose and ensured that the pilot project was successfully completed. These project coordinators were, in turn, supported by the national management team and were given opportunities to communicate and share experiences among themselves. This system of support proved to be very effective at both the local and national levels.

\section{Flexibility}

The ability to respond to a changing environment and to make appropriate adjustments is critical to the success of any project. Timetables needed to be adjusted and resources reallocated to respond to unanticipated demands. 


\section{Sustainability}

For participants, the value of the project was reinforced by the knowledge that there was a commitment to see individual projects continue beyond the life of the RTA initiative.

\section{The RTA Legacy}

In 2009, the CNA argued that reversing the nursing shortage required the implementation of several "tested solutions," including increasing nurse productivity, improving retention, reducing the attrition of new nurses and reducing absenteeism (Tomblin Murphy et al. 2009). The RTA projects have begun to put these solutions to work, with the hope of contributing to a robust nursing workforce that meets the needs of Canadians. The RTA initiative was designed to involve staff nurses in the research, policy and oversight of these projects. Their insights helped the projects reflect the realities of the workplace and propose appropriate responses.

The Research to Action initiative concluded in March 2011, but the innovative workplace strategies implemented through the individual projects have continued, although in some cases they have been modified:

- Newfoundland and Labrador has modified the 80/20 model to a possible $85 / 15$ or $90 / 10$ model of professional development, and plans to implement the model in other facilities in the health region.

- A partnership between employers and the Faculty of Nursing at the University of Prince Edward Island developed a proposal to establish an ongoing PEI-based critical care and emergency nursing program utilizing 80/20 staffing models and mentorship. The program was approved to begin in January 2012.

- Nova Scotia now has a province-wide electronic employment placement system for its graduating nurses. The project also enabled the development of new tools and resources to support mentorship workshops.

- Mentorship guides and an electronic orientation program are now available in French. The mentorship program is being expanded to all four zones of the Vitalité Health Network in New Brunswick.

- Staffing tools developed in Ontario and Saskatchewan continue to be refined and used within their respective health regions. Another facility in Saskatchewan will begin using the staffing tool on a unit in 2012 .

- The Manitoba government has provided funding to implement the enhanced orientation to long-term care program across the province. 
- The Prince Edward Island Department of Health and Wellness is now using the Manitoba orientation program in all of the province's public and private long-term care facilities.

- Several protocols developed through the 80/20 project in Kamloops, British Columbia have been adopted throughout the Interior Health Region.

- Critical care training for nurses at Qikiqtani General Hospital (QGH) in Iqaluit, Nunavut enhanced the leadership and training skills for front-line nurses. Critical care policies and procedures similar to those of the Ottawa Hospital (which receives patients from QGH) have been developed and adopted. These enhancements will result in improved patient care for northern patients at both hospitals.

- The mentorship program developed in Iqaluit will serve as the model for future work in Nunavut.

- The results of the Alberta evaluation have been used to inform decisionmaking in ongoing collective bargaining retention and recruitment initiatives.

- The RTA evaluations have contributed to the national body of evidence-based research on retention and recruitment approaches.

- National partners continue to work together on nursing issues.

\section{Conclusion}

A positive work environment is crucial to the retention and recruitment of nurses. It takes more than 24 months to change a workplace culture. Nevertheless, the evaluations have shown that the Research to Action initiative has made a difference. Nurses who participated in the 10 projects feel they can bring about change in their workplaces. They have greater confidence, an increased commitment to their profession and a renewed sense of purpose.

The RTA initiative offered nurses an opportunity to acquire new skills and new competencies and to share what they learned with their colleagues. This visible commitment to professional development has led to improved morale. It has helped to renew professional commitment and has kept nurses from leaving the profession.

The RTA initiative demonstrated that change in the workplace is possible if the resolve, resources and partnerships needed to improve circumstances are in place. However, change on a larger scale is needed, and more areas of nursing need attention, including rural and remote nursing, community nursing and mental health. With buy-in from key stakeholders, and the will to effect change, we can improve nursing at the systemic level for the benefit of employers, nurses and patients. 


\section{References}

Aiken, L., S.P. Clarke, D.M. Sloane, J. Sochalski and J.H. Silber. 2002. "Hospital Nurse Staffing and Patient Mortality, Nurse Burnout and Job Dissatisfaction." Journal of the American Medical Association 288(16): 1987-93. doi:10.1001/jama.288.16.1987.

Canadian Federation of Nurses Unions (CFNU). 2011. “Trends in Own Illness or DisabilityRelated Absenteeism and Overtime among Publicly Employed Registered Nurses: Quick Facts.” Ottawa: Informetrica Limited.

Curley, M.A.Q. 2007. Synergy: The Unique Relationship Between Nurses and Patients. Indianapolis: Sigma Theta Tau International.

Lasota, M. 2009. Trends in Own Illness or Disability-Related Absenteeism and Overtime among Publicly Employed Registered Nurses. Summary of Key Findings. Ottawa: Canadian Federation of Nurses Unions.

O’Brien-Pallas, L., G. Tomblin Murphy and J. Shamian. 2008. Understanding the Costs and Outcomes of Nurses' Turnover in Canadian Hospitals. Nursing Turnover Study FRN\#66350. Toronto: Nursing Health Services Research Unit, University of Toronto.

Tomblin Murphy, G., S. Birch, R. Alder, A. MacKenzie, L. Lethbridge, L. Little and A. Cook. 2009. Tested Solutions for Eliminating Canada's Registered Nurse Shortage. Ottawa: Canadian Nurses Association. 